\title{
Compactness of certain class of singular minimal hypersurfaces
}

\author{
Akashdeep Dey *
}

\begin{abstract}
Given a closed Riemannian manifold $\left(N^{n+1}, g\right), n+1 \geq 3$ we prove the compactness of the space of singular, minimal hypersurfaces in $N$ whose volumes are uniformly bounded from above and the $p$-th Jacobi eigenvalue $\lambda_{p}$ 's are uniformly bounded from below. This generalizes the results of Sharp Sha17] and Ambrozio-Carlotto-Sharp ACS16] in higher dimensions.
\end{abstract}

\section{Introduction}

A hypersurface of a Riemannian manifold $\left(N^{n+1}, g\right)$ is called minimal if it is a critical point of the $n$-dimensional area functional. By the combined works of Almgren Alm65, Pitts Pit81 and Schoen-Simon SS81, one gets the following Theorem.

Theorem 1.1 ([Alm65], Pit81, SS81]). Let $\left(N^{n+1}, g\right)$ be an arbitrary closed Riemannian manifold with $n+1 \geq 3$. Then $N$ contains a singular, minimal hypersurface which is smooth and embedded outside a singular set of Hausdorff dimension atmost $n-7$. In particular, when $3 \leq n+1 \leq 7$ there exists a smooth, closed, embedded, minimal hypersurface in $N$.

Recently, Almgren-Pitts min-max theory has been further developed to show that minimal hypersurfaces exist in abundance when the ambient dimension $3 \leq n+1 \leq 7$. By the results of Marques-Neves MN17 and Song Son18 every closed Riemannian manifold $N$ of dimension $3 \leq n+1 \leq 7$ contains infinitely many minimal hypersurfaces. Moreover, Irie, Marques and Neves have shown that [MN18] for a generic metric the union of all closed, minimal hypersurfaces is dense in $N$; this theorem was later improved by Marques, Neves and Song in MNS17] where they proved that for a generic metric there exists an equidistributed sequence of closed, minimal hypersufaces in $N$. The Weyl law for the volume spectrum proved by Liokumovich, Marques and Neves [LMN18] played a major role in the arguments of [MN18, and MNS17. There is yet another proof of the existence of infinitely many closed, minimal hypersurfaces for a generic metric on $N$ which follows form the papers by Marques-Neves MN17 and Zhou [Zho19]. The reason of the upper bound of the dimension $n+1 \leq 7$

*Email: adey@math.princeton.edu, dey.akash01@gmail.com 
in Son18, IMN18, MNS17, Zho19 is that the space of singular, minimal hypersurfaces is not well understood unlike the smooth case (Whi91, [Whi17]).

Using the Allen-Cahn equation Chodosh and Mantoulidis [CM18 have proved the existence of infinitely many minimal surfaces for generic metrics in dimension 3; Gaspar and Guaraco GG18 have given alternative proofs of the above mentioned density and equidistribution theorems.

In higher dimensions, Li [Li19] has proved that a closed manifold $M^{n+1}, n+1 \geq$ 8 equipped with a generic Riemannian metric contains infinitely many singular, minimal hypersurfaces with optimal regularity (i.e. the singular set has Hausdorff dimension atmost $n-7$ ).

One of the key ingredients of the papers [Son18, [MN18, [MNS17, Zho19] is Sharp's compactness theorem [Sha17] which asserts certain compactness properties of the set of smooth, closed, minimal hypersurfaces in a Riemannian manifold $\left(M^{n+1}, g\right), 3 \leq n+1 \leq 7$ with bounded volume and index. This result was generalized by Ambrozio, Carlotto and Sharp ACS16 where instead of bounded volume and index, an upper bound of the volume and a lower bound of the $p$-th Jacobi eigenvalue $\lambda_{p}$ (for some $p \in \mathbb{N}$ ) was assumed. (We note that for a smooth, closed minimal hypersurface $\Sigma, \operatorname{Ind}(\Sigma) \leq I$ is equivalent to $\lambda_{I+1}(\Sigma) \geq 0$.)

In the present article we will suitably generalize the results of [Sha17 and ACS16 in higher dimensions; for that, we need to consider the minimal hypersurfaces which may have singularities. We will state the notion of the index and the $p$-th Jacobi eigenvalue for a stationary $n$-varifold and prove the following Theorem.

Theorem 1.2. Let $\left\{M_{k}\right\}_{k=1}^{\infty}$ be a sequence of closed, connected, singular, minimal hypersurfaces in a closed Riemannian manifold $\left(N^{n+1}, g\right), n+1 \geq 3$. Let $V_{k}=\left|M_{k}\right|$, the varifold associated to $M_{k}$. Suppose, there exist $\Lambda>0, \alpha \geq 0$, $p \in \mathbb{N}$ such that for all $k$

- $\mathcal{H}^{n-2}\left(\operatorname{sing}\left(M_{k}\right)\right)=0$

- $\mathcal{H}^{n}\left(M_{k}\right)=\left\|V_{k}\right\|(N) \leq \Lambda$

- $\lambda_{p}\left(V_{k}\right) \geq-\alpha$

Then there is a stationary, integral varifold $V$ such that possibly after passing to a subsequence, $V_{k} \longrightarrow V$ in the $\mathbf{F}$ metric. Moreover, denoting $M=\operatorname{spt}(V)$ we have

- $\|V\|(N) \leq \Lambda$

- $\lambda_{p}(V) \geq-\alpha$

- $\mathcal{H}^{s}(\operatorname{sing}(M))=0 \quad \forall s>n-7$

- The convergence is smooth and graphical over the compact subsets of reg $(M) \backslash \mathcal{Y}$ where $\mathcal{Y}$ is a finite subset of reg $(M)$ with $|\mathcal{Y}| \leq p-1$.

From the definitions of the index and the Jacobi eigenvalue, it will be clear that 
Ind $(V) \leq I$ if and only if $\lambda_{I+1}(V) \geq 0$. Therefore, Theorem 1.2 has the following Theorem as a corollary which generalizes Sharp's compactness theorem Sha17] in higher dimensions.

Theorem 1.3. Let $\left\{M_{k}\right\}_{k=1}^{\infty}$ be a sequence of closed, connected, singular, minimal hypersurfaces in a closed Riemannian manifold $\left(N^{n+1}, g\right), n+1 \geq 3$. Suppose for all $k, \mathcal{H}^{n-2}\left(\operatorname{sing}\left(M_{k}\right)\right)=0, \mathcal{H}^{n}\left(M_{k}\right) \leq \Lambda$ and $\operatorname{Ind}\left(\left|M_{k}\right|\right) \leq I$. Then there is a stationary, integral varifold $V$ such that possibly after passing to a subsequence, $\left|M_{k}\right| \longrightarrow V$ in the $\mathbf{F}$ metric, $\|V\|(N) \leq \Lambda$ and $\operatorname{Ind}(V) \leq I$. Further, if $M=\operatorname{spt}(V)$ then $\mathcal{H}^{s}(\operatorname{sing}(M))=0 \quad \forall s>n-7$ and the convergence is smooth and graphical over the compact subsets of reg $(M) \backslash \mathcal{Y}$ where $\mathcal{Y}$ is a finite subset of $\operatorname{reg}(M)$ with $|\mathcal{Y}| \leq I$.

The space of minimal hypersurfaces with bounded volume and index is particularly interesting; due to the work of Marques and Neves [MN16], the minimal hypersurfaces constructed by the min-max procedure have bounded volume and index. More precisely, they have proved the following Theorem.

Theorem 1.4 ([MN16]). Suppose $\left(N^{n+1}, g\right)$ is a closed Riemannian manifold, $n+1 \geq 3$. Let $X$ be an $m$ dimensional simplicial complex and $\Pi$ be a $\mathcal{F}$-homotopy class of continuous maps from $X$ to $\mathcal{Z}_{n}\left(N ; \mathbf{F} ; \mathbb{Z}_{2}\right)$. We define

$$
\mathbf{L}(\Pi)=\inf _{\Phi \in \Pi} \sup _{x \in X}\{\mathbf{M}(\Phi(x))\}
$$

Then there is a stationary, integral varifold $V$ with $\operatorname{spt}(V)=\Sigma$ such that

- $\|V\|(N)=\mathbf{L}(\Pi)$

- $\operatorname{Ind}(V) \leq m$

- $\mathcal{H}^{s}(\operatorname{sing}(\Sigma))=0 \quad \forall s>n-7$.

The index upper bound of the minimal hypersurfaces in the Allen-Cahn settings has been proved by Gaspar Gas17] and Hiesmayr Hie17.

If we take $M_{k}$ to be $M$ for all $k$ in Theorem 1.2, we get the following regularity result.

Proposition 1.5. Let $M^{n}$ be a singular, minimal hypersurface in $\left(N^{n+1}, g\right)$, $n+1 \geq 3$. Suppose, $\mathcal{H}^{n-2}(\operatorname{sing}(M))=0$ and $\lambda_{p}(|M|)>-\infty$ for some $p$. Then $\mathcal{H}^{s}(\operatorname{sing}(M))=0 \forall s>n-7$.

The proof of Theorem 1.2 is very similar to that of [Sha17] and ACS16]. However, for the sake of completeness we will give a self-contained proof of it.

Acknowledgements. I am very grateful to my advisor Prof. Fernando Codá Marques for many helpful discussions and for his support and guidance. I also thank Yangyang Li and Antoine Song for answering some of my questions. The author is partially supported by NSF grant DMS-1811840. 


\section{Notations and Preliminaries}

\section{$2.1 \quad$ Notations}

Here we summarize the notations which will be frequently used later.

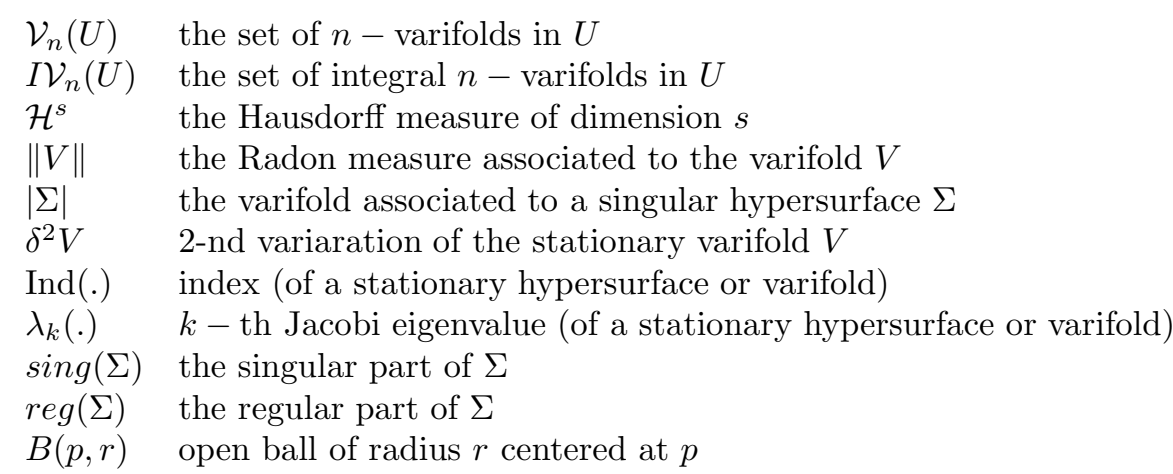

\subsection{Preliminaries from geometric measure theory}

Here we will briefly discuss the notion of varifold and various related concepts; further details can be found in Simon's book [Sim].

Given a Riemannian manifold $\left(U^{n+1}, g\right)$ let $G_{k}(U)$ denote the Grassmanian bundle of $k$-dimensional hyperplanes over $U$. A $k$-varifold in $U$ is a positive Radon measure on $G_{k}(U)$. The topology on the space of $k$-varifolds $\mathcal{V}_{k}(U)$ is given by the weak* topology i.e. a net $\left\{V_{i}\right\}_{i \in I} \subset \mathcal{V}_{k}(U)$ converges to $V$ iff

$$
\int_{G_{k}(U)} f(x, \omega) d V_{i}(x, \omega) \longrightarrow \int_{G_{k}(U)} f(x, \omega) d V(x, \omega)
$$

for all $f \in C_{c}\left(G_{k}(U)\right)$. This topology is metrizable and the metric is denoted by $\mathbf{F}$. If $V \in \mathcal{V}_{k}(U)$ and $\pi: G_{k}(U) \longrightarrow U$ denotes the canonical projection then $\|V\|=\pi_{*} V$ is a Radon measure on $U ;\|V\|(A)=V\left(\pi^{-1}(A)\right)$.

If $\varphi: U \longrightarrow U^{\prime}$ is a diffeomorphism and $V \in \mathcal{V}_{k}(U)$, we define $\varphi_{*} V \in \mathcal{V}_{k}\left(U^{\prime}\right)$ by the following formula

$$
\left(\varphi_{*} V\right)(g)=\int_{G_{k}(U)} g\left(\varphi(x), d_{x} \varphi(\omega)\right) J \varphi(x, \omega) d V(x, \omega)
$$

where

$$
J \varphi(x, \omega)=\left(\operatorname{det}\left(\left(\left.d_{x} \varphi\right|_{\omega}\right)^{t} \circ\left(\left.d_{x} \varphi\right|_{\omega}\right)\right)\right)^{1 / 2}
$$

is the Jacobian factor and $g \in C_{c}\left(G_{k}\left(U^{\prime}\right)\right)$. Given a compactly supported, smooth vector-field $X$ on $U$ let $\varphi_{t}$ denote the flow of $X$; the first variation and second variation of $V$ are given by 


$$
\delta V(X)=\left.\frac{d}{d t}\right|_{0}\left\|\left(\varphi_{t}\right)_{*} V\right\|(U) \quad ; \quad \delta^{2} V(X, X)=\left.\frac{d^{2}}{d t^{2}}\right|_{0}\left\|\left(\varphi_{t}\right)_{*} V\right\|(U)
$$

We say that $V$ is stationary if $\delta V(X)=0$ for all $X$ and a stationary varifold $V$ is called stable if $\delta^{2} V(X, X) \geq 0$ for all $X$.

Given a $k$-rectifiable set $S \subset U$ and a non-negative function $\theta \in L_{l o c}^{1}\left(S, \mathcal{H}^{k}\llcorner S)\right.$ we define the $k$-varifold $\mathbf{v}(S, \theta)$ by

$$
\mathbf{v}(S, \theta)(f)=\int_{S} f\left(x, T_{x} S\right) \theta(x) d \mathcal{H}^{k}(x)
$$

where $T_{x} S$ denotes the tangent space of $S$ at $x$ which exists $\mathcal{H}^{k}\llcorner S$-a.e. $V$ is called an integral $k$-varifold if $V=\mathbf{v}(S, \theta)$ for some $S$ and $\theta$ with $\theta$ taking non-negative integer values $\mathcal{H}^{k}\llcorner S$-a.e.

In the present article we will only deal with $n$-varifolds and from now on we will simply write 'varifold' instead of ' $n$-varifold'. Given $A \subset U$ we define the regular and singular part of $A$

$$
\begin{aligned}
\operatorname{reg}(A)=\{x \in A: & \exists \text { open } P \subset U \text { containing } x \text { such that } \\
& P \cap A \text { is a smooth, embedded hypersurface }\}
\end{aligned}
$$

and

$$
\operatorname{sing}(A)=A \backslash \operatorname{reg}(A) .
$$

Further, by a singular, minimal hypersurface $\Sigma$ in $U$ we will mean that $\Sigma \subset U$ is a closed, $n$-rectifiable set with $\mathcal{H}^{n-1}(\operatorname{sing}(\Sigma))=0$ and $|\Sigma|=\mathbf{v}\left(\Sigma, \mathbf{1}_{\Sigma}\right)$ (where $\mathbf{1}_{\Sigma}$ is the constant function 1) is stationary. By [Im96] (Equation (4)), $|\Sigma|$ is stationary in $U$ if and only if $\operatorname{reg}(\Sigma)$ is a smooth, minimal hypersurface and $\mathcal{H}^{n}(\Sigma \cap B(x, r)) \leq C\left(U^{\prime}\right) r^{n}$ for all $B(x, r) \subseteq U^{\prime} \subset \subset U$.

\section{Index and Jacobi eigenvalues of a stationary varifold}

We will now state the notion of the index and the Jacobi eigenvalues of a stationary varifold following the paper by Marques and Neves [MN16. The definition is motivated by the following min-max characterization of $\lambda_{k}(\Sigma)$ when $\Sigma^{n} \subset\left(U^{n+1}, g\right)$ is a smooth, minimal hypersurface.

$$
\lambda_{k}(\Sigma)=\inf _{\operatorname{dim}(V)=k} \sup _{X \in V \backslash\{0\}}\left(\frac{\delta^{2} \Sigma(X, X)}{\int_{\Sigma}|X|^{2} d \mathcal{H}^{n}}\right)
$$

The infimum is over all the $k$-dimensional linear subspaces $V \subset \Gamma_{c}(N \Sigma)=$ compactly supported smooth sections of $N \Sigma$. Therefore, $\lambda_{k}(\Sigma)<a$ iff there is a $k$-dimensional subspace $V \subset \Gamma_{c}(N \Sigma)$ such that for all $X \in V \backslash\{0\}$,

$$
\delta^{2} \Sigma(X, X)<a \int_{\Sigma}|X|^{2} d \mathcal{H}^{n}
$$


Given a Riemannian manifold $\left(U^{n+1}, g\right)$ and $k \in \mathbb{N}(0 \notin \mathbb{N})$, a k-parameter family of diffeomorphisms is a smooth map $F: \bar{B}^{k}(0,1)\left(\subset \mathbb{R}^{k}\right) \longrightarrow \operatorname{Diff}(U)$ such that

- $F_{v}(=F(v))=\left(F_{-v}\right)^{-1} \forall v \in \bar{B}^{k}(0,1)$ and $F_{0}=\mathrm{Id}$

- there exists open $U^{\prime} \subset \subset U$ such that $\left.F_{v}\right|_{U \backslash U^{\prime}}=\operatorname{Id} \forall v \in \bar{B}^{k}(0,1)$

If $F$ is a $k$-parameter family of diffeomorphisms, we define the vector-fields $Y_{i}$, $i=1, \ldots, k$ by

$$
\left.Y_{i}\right|_{p}=\left.\frac{d}{d t}\right|_{0} F_{t e_{i}}(p)
$$

Suppose further, we have a stationary varifold $V$ in $U$. Then we define a smooth function $A^{V}$ and a quadratic form $K^{V}$ as follows.

$$
\begin{gathered}
A^{V}: \bar{B}^{k}(0,1) \longrightarrow[0, \infty), \quad A^{V}(v)=\left\|\left(F_{v}\right)_{\#} V\right\|(U) \\
K^{V}: \mathbb{R}^{k} \times \mathbb{R}^{k} \longrightarrow \mathbb{R}, \quad K^{V}(u, u)=\left\|\sum_{i} u_{i} Y_{i}\right\|_{L^{2}(U,\|V\|)}^{2}
\end{gathered}
$$

Remark 3.1. If $V_{i} \longrightarrow V$ in the $\mathbf{F}$ metric then $A^{V_{i}} \longrightarrow A^{V}$ ([Pit81], Section 2.3) in the smooth topology and also $K^{V_{i}} \longrightarrow K^{V}$ smoothly on compact subsets.

Definition 3.2. Given a stationary varifold $V$ in $U, k \in \mathbb{N}$ and $\alpha \geq 0$ we say that $\lambda_{k}(V)<-\alpha$ if there exists a $k$-parameter family of diffeomorphisms $F$ such that

$$
\left.D^{2} A^{V}\right|_{0}(u, u)<-\alpha K^{V}(u, u)
$$

for all $u \in \mathbb{R}^{k} \backslash\{0\}$ or equivalently for all $u \in \mathbb{R}^{k}$ with $\|u\|=1$. Further, by $\lambda_{k}(V) \geq-\alpha$ we will mean that $\lambda_{k}(V)<-\alpha$ does not hold. If $\lambda_{k}(V)<-\alpha$ then restricting $F$ to $\bar{B}^{k-1}(0,1) \subset \bar{B}^{k}(0,1)$ we get that $\lambda_{k-1}(V)<-\alpha$ as well. Therefore, it will be natural to define

$$
\operatorname{Ind}(V)= \begin{cases}0 & \text { if }\left\{I \in \mathbb{N}: \lambda_{I}(V)<0\right\}=\emptyset \\ \sup \left\{I \in \mathbb{N}: \lambda_{I}(V)<0\right\} & \text { otherwise. }\end{cases}
$$

Hence, $\operatorname{Ind}(V) \leq I$ is equivalent to $\lambda_{I+1}(V) \geq 0$. Further, $\operatorname{Ind}(V)=0$ iff $\lambda_{1}(V) \geq 0$ iff $V$ is stable.

Remark 3.3. By Remark 3.1 and from the above definition it is clear that whenever $\lambda_{k}(V)<-\alpha$ and $\mathbf{F}\left(V, V^{\prime}\right)$ is sufficiently small, we have $\lambda_{k}\left(V^{\prime}\right)<-\alpha$ as well.

Proposition 3.4. Given $\Lambda>0, k \in \mathbb{N}$ and $\alpha \geq 0$, the following sets are compact with respect to the $\mathbf{F}$ metric topology.

$\mathcal{M}_{U}(\Lambda, k, \alpha)=\left\{V \in \mathcal{V}_{n}(U): V\right.$ is stationary, $\|V\| \leq \Lambda$ and $\left.\lambda_{k}(V) \geq-\alpha\right\} \subset \mathcal{V}_{n}(U)$

$\mathcal{M}_{U}^{\prime}(\Lambda, k, \alpha)=\left\{V \in I \mathcal{V}_{n}(U): V\right.$ is stationary, $\|V\| \leq \Lambda$ and $\left.\lambda_{k}(V) \geq-\alpha\right\} \subset I \mathcal{V}_{n}(U)$ 
Proof. By the standard compactness theorems, if $\left\{V_{i}\right\}_{i=1}^{\infty}$ is a sequence of stationary varifolds with $\left\|V_{i}\right\| \leq \Lambda$ then upto a subsequence $V_{i}$ converges to a stationary varifold $V$ in the $\mathbf{F}$ metric with $\|V\| \leq \Lambda$. Further, by Allard's theorem All72] if $V_{i}$ 's are integral varifolds, $V$ is also an integral varifold. Moreover, by Remark 3.3 if $\lambda_{k}(V)<-\alpha$ then for $i$ large $\lambda_{k}\left(V_{i}\right)<-\alpha$ as well. Hence, $\lambda_{k}(V) \geq-\alpha$.

Theorem 3.5. Let $\Sigma$ be a singular, minimal hypersurface in $U, V=|\Sigma|$ and $\mathcal{H}^{n-2}(\operatorname{sing}(\Sigma))=0$. Then, $\lambda_{k}(V)<-\alpha \leq 0 \Longleftrightarrow \lambda_{k}(\operatorname{reg}(\Sigma))<-\alpha$. Hence, $\operatorname{Ind}(V)=\operatorname{Ind}($ reg $(\Sigma))$; therefore, $V$ is stable iff reg $(\Sigma)$ is stable.

Proof. We note that

$$
\left.D^{2} A^{V}\right|_{0}(u, u)=\left.\frac{d^{2}}{d t^{2}}\right|_{0} A^{V}(t u, t u)=\left.\frac{d^{2}}{d t^{2}}\right|_{0}\left\|\left(F_{t u}\right)_{\#} V\right\|(U)=\delta^{2} V\left(\sum_{i} u_{i} Y_{i}, \sum_{i} u_{i} Y_{i}\right)
$$

Let $\lambda_{k}(\operatorname{reg}(\Sigma))<-\alpha$. Then there are $k$ linearly independent, compactly supported normal vector-fields on $\operatorname{reg}(\Sigma)$ say $X_{1}, X_{2}, \ldots, X_{k}$ such that

$$
\delta^{2} V(X, X)<-\alpha\|X\|_{L^{2}(\Sigma)}^{2}
$$

for any non-zero vector-field $X$ in the span of $\left\{X_{i}\right\}_{i=1}^{k}$. We extend each $X_{i}$ to a compactly supported, globally defined vector-field on $U$ and continue to call it by $X_{i}$; we define $F_{v}=\Phi^{\sum_{i} v_{i} X_{i}}$ where $\Phi^{X}$ denotes the time 1 flow of the vector-field $X$. Let us check that this choice of $F$ indeed works. Clearly, $F_{-v}=F_{v}^{-1}$.

By 3.1

$$
\left.D^{2} A^{V}\right|_{0}(u, u)=\delta^{2} V\left(\sum_{i} u_{i} X_{i}, \sum_{i} u_{i} X_{i}\right)<-\alpha\left\|\sum_{i} u_{i} X_{i}\right\|_{L^{2}(\Sigma)}^{2}
$$

For the converse, we consider the following.

$\delta^{2} V(X, X)=\int_{\Sigma}\left(\left(\operatorname{div}_{\Sigma} X\right)^{2}+\sum_{i=1}^{n}\left|\left(\nabla_{\tau_{i}} X\right)^{\perp}\right|^{2}-\sum_{i, j=1}^{n}\left\langle\tau_{i}, \nabla_{\tau_{j}} X\right\rangle\left\langle\tau_{j}, \nabla_{\tau_{i}} X\right\rangle\right) d \mathcal{H}^{n}$

where $\left\{\tau_{i}\right\}_{i=1}^{n}$ is an orthonormal basis of $T_{x} \Sigma$. Since $\mathcal{H}^{n-2}(\operatorname{sing}(\Sigma))=0$, given any $\delta>0$ and $0<\kappa<1$ we can choose balls $\left\{B\left(x_{i}, r_{i}\right)\right\}_{i=1}^{K}$ such that each $r_{i}<\kappa, \operatorname{sing}(\Sigma) \subset \cup_{i} B\left(x_{i}, r_{i}\right)$ and $\sum_{i} r_{i}^{n-2}<\delta$. Therefore, $\sum_{i} r_{i}^{n-1}<\delta$ and $\sum_{i} r_{i}^{n}<\delta$ as well. We choose smooth cut-off functions $0 \leq \zeta_{i} \leq 1$ on $U$ such that

$$
\zeta_{i}= \begin{cases}0 & \text { on } B\left(x_{i}, r_{i}\right) \\ 1 & \text { outside } B\left(x_{i}, 2 r_{i}\right)\end{cases}
$$


and $\left|\nabla \zeta_{i}\right| \leq 2 / r_{i}$ (this can be ensured by choosing $\kappa$ sufficiently small). Let $\zeta_{\delta}=\min _{i} \zeta_{i}$. From the second variation formula, we see that 1

$$
\left|\delta^{2} V(X, X)-\delta^{2} V\left(\zeta_{\delta} X, \zeta_{\delta} X\right)\right| \leq \int_{\Sigma}\left(\left(1-\zeta_{\delta}^{2}\right)+\left|\nabla \zeta_{\delta}\right|+\left|\nabla \zeta_{\delta}\right|^{2}\right) f(X, \nabla X) d \mathcal{H}^{n}
$$

where $f$ is an expression involving $X$ and $\nabla X$. By the monotonicity formula, the R.H.S. of this equation is bounded by $C \delta$ for some constant $C$ depending only on $(U, g), n,\|V\|(U)$ and $\|X\|_{C^{1}}$.

Therefor, for $u \in \mathbb{R}^{k}$ with $\|u\|=1$

$$
\begin{aligned}
\delta^{2} V\left(\zeta_{\delta} \sum_{i} u_{i} Y_{i}^{\perp}, \zeta_{\delta} \sum_{i} u_{i} Y_{i}^{\perp}\right) & =\delta^{2} V\left(\zeta_{\delta} \sum_{i} u_{i} Y_{i}, \zeta_{\delta} \sum_{i} u_{i} Y_{i}\right) \\
& \leq \delta^{2} V\left(\sum_{i} u_{i} Y_{i}, \sum_{i} u_{i} Y_{i}\right)+C \delta
\end{aligned}
$$

Here $C$ depends only on $(U, g), n,\|V\|(U)$ and $\left\|Y_{i}\right\|_{C^{1}}$ and not on $u$.

We assume $\lambda_{k}(V)<-\alpha$. By Definition 3.2 and equation 3.1 for all $u \in \mathbb{R}^{k}$ with $\|u\|=1$,

$$
\delta^{2} V\left(\sum_{i} u_{i} Y_{i}, \sum_{i} u_{i} Y_{i}\right)<-\alpha\left\|\sum_{i} u_{i} Y_{i}\right\|_{L^{2}(\Sigma)}^{2} \Longrightarrow \sum_{i} u_{i} Y_{i} \neq 0
$$

Therefore,

$$
\sup _{\|u\|=1} \frac{\delta^{2} V\left(\sum_{i} u_{i} Y_{i}, \sum_{i} u_{i} Y_{i}\right)}{\left\|\sum_{i} u_{i} Y_{i}\right\|_{L^{2}}^{2}} \leq-\alpha-2 \varepsilon
$$

for some $\varepsilon>0$. Hence, using 3.4 for all $\|u\|=1$ and $\delta$ sufficiently small

$$
\begin{aligned}
& \delta^{2} V\left(\zeta_{\delta} \sum_{i} u_{i} Y_{i}^{\perp}, \zeta_{\delta} \sum_{i} u_{i} Y_{i}^{\perp}\right) \\
& \leq \delta^{2} V\left(\sum_{i} u_{i} Y_{i}, \sum_{i} u_{i} Y_{i}\right)+C \delta \\
& \leq(-\alpha-2 \varepsilon) \int_{\Sigma}\left|\sum_{i} u_{i} Y_{i}\right|^{2} d \mathcal{H}^{n}+C \delta \\
& \leq(-\alpha-2 \varepsilon) \int_{\Sigma}\left|\sum_{i} u_{i} Y_{i} \zeta_{\delta}\right|^{2} d \mathcal{H}^{n}+C \delta \\
& <(-\alpha-\varepsilon) \int_{\Sigma}\left|\sum_{i} u_{i} Y_{i} \zeta_{\delta}\right|^{2} d \mathcal{H}^{n} \\
& \leq(-\alpha-\varepsilon)\left\|\sum_{i} u_{i} Y_{i}^{\perp} \zeta_{\delta}\right\|_{L^{2}(\Sigma)}^{2}
\end{aligned}
$$

\footnotetext{
${ }^{1}$ Though $\zeta_{\delta}$ is only a Lipschitz continuous function, its use in the subsequent calculations can be justified by an approximation argument.
} 
To justify [3.7, we observe:

$$
\begin{aligned}
& \int_{\Sigma}\left|\sum_{i} u_{i} Y_{i} \zeta_{\delta}\right|^{2} d \mathcal{H}^{n}=\int_{\Sigma}\left|\sum_{i} u_{i} Y_{i}\right|^{2} d \mathcal{H}^{n}+\int_{\Sigma}\left(\zeta_{\delta}^{2}-1\right)\left|\sum_{i} u_{i} Y_{i}\right|^{2} d \mathcal{H}^{n} \\
& \geq \inf _{\|u\|=1} \int_{\Sigma}\left|\sum_{i} u_{i} Y_{i}\right|^{2} d \mathcal{H}^{n}-C \sum_{i} r_{i}^{n} \text { (using monotonicity formula) } \\
& \geq \theta-C \delta \text { (for some } \theta>0 \text { by } 3.5 \text { and } C \text { is independent of } u \text { ) }
\end{aligned}
$$

Clearly 3.8 implies that $\left\{\zeta_{\delta} Y_{i}^{\perp}\right\}_{i=1}^{k}$ are linearly independent normal vector fields on $\operatorname{reg}(\Sigma)$ and $\lambda_{k}(\operatorname{reg}(\Sigma))<-\alpha$

In view of the above Theorem 3.5, we will use the terms $\lambda_{k}(V)$ and $\lambda_{k}(r e g(\Sigma))$ interchangeably.

\section{Modifications of the results of Schoen-Simon SS81}

Suppose the unit ball $B^{n+1}(0,1) \subset \mathbb{R}^{n+1}$ is equipped with a Riemannian metric $g ; \mu_{1}$ is a constant such that if $g=g_{i j} d x^{i} d x^{j}$

$$
\sup _{B^{n+1}(0,1)}\left|\frac{\partial g_{i j}}{\partial x_{k}}\right| \leq \mu_{1} \quad \sup _{B^{n+1}(0,1)}\left|\frac{\partial^{2} g_{i j}}{\partial x_{k} \partial x_{l}}\right| \leq \mu_{1}^{2}
$$

Theorem 4.1 (Modification of Schoen-Simon SS81, Theorem 1 ; page 747). Suppose $\Sigma$ is a singular, minimal hypersurface in $\left(B^{n+1}(0,1), g\right)$ satisfying $\mathcal{H}^{n-2}(\operatorname{sing}(\Sigma))=0, \mathcal{H}^{n}(\Sigma) \leq \mu$ and $\lambda_{1}(|\Sigma|) \geq-\alpha$ for some $\alpha \geq 0$. Then there exist $\delta_{0} \in(0,1), r_{0} \in(0,1 / 4)$ and $c>0$ depending only on $n, \mu, \mu_{1}, \alpha$ such that the following holds. If $x=\left(x^{\prime}, x_{n+1}\right) \in \Sigma \cap B^{n+1}(0,1 / 4), \rho \leq r_{0}, \Sigma^{\prime}$ is the connected component of $\Sigma \cap C(x, \rho)\left(C(x, \rho)\right.$ is the cylinder on $\left.B^{n}(x, \rho)\right)$ containing $x$ and

$$
\sup _{y=\left(y^{\prime}, y_{n+1}\right) \in \Sigma^{\prime}}\left|y_{n+1}-x_{n+1}\right| \leq \delta_{0} \rho
$$

then, $\Sigma^{\prime} \cap C(x, \rho / 2)$ consists of disjoint union of graphs of functions $u_{1}<u_{2}<$ $\ldots<u_{k}$ defined on $B^{n}(x, \rho / 2)$ satisfying the following estimate.

$$
\sup _{B^{n}\left(x, \frac{\rho}{2}\right)}\left(\left|D u_{i}\right|+\rho\left|D D u_{i}\right|\right) \leq c \delta_{0}
$$

for $i=1,2, \ldots, k$. In particular, $\Sigma$ is smooth near $x$ and the second fundamental form $\left|A_{\Sigma}(x)\right| \leq c / \rho$ (for possibly a different constant $c$ ). 
Remark 4.2. The difference between the above Theorem 4.1 and Theorem 1 of Schoen-Simon SS81 is that instead of assuming $|\Sigma|$ is stable we have assumed that $\lambda_{1}(|\Sigma|) \geq-\alpha$. Indeed under this weaker assumption, the stability inequality (1.17) of Schoen-Simon [SS81] (page 746) continues to hold with the constant $c_{5}$ replaced by $c_{5}+\frac{\alpha}{\mu_{1}^{2}}$; therefore all the successive calculations in the paper [SS81] go through.

Theorem 4.3 (Modification of Schoen-Simon SS81, Theorem 2; page 784). Let $\left\{\Sigma_{q}\right\}$ be a sequence of singular, minimal hypersurfaces in $\left(B^{n+1}(0,1), g\right)$ such that $\mathcal{H}^{n-2}\left(\operatorname{sing}\left(\Sigma_{q}\right)\right)=0, \lambda_{1}\left(\left|\Sigma_{q}\right|\right) \geq-\alpha$ and $\left|\Sigma_{q}\right|$ converges to a varifold $W ; 0 \in \operatorname{spt}(W)=\Sigma$. Then $\mathcal{H}^{s}\left(\operatorname{sing}(\Sigma) \cap B^{n+1}\left(0, \frac{1}{2}\right)\right)=0$ for all $s>n-7$.

Proof. As before, The difference between the above Theorem 4.3 and Theorem 2 of Schoen-Simon [SS81] is that instead of assuming $|\Sigma|$ is stable we have assumed that $\lambda_{1}(|\Sigma|) \geq-\alpha$.

The proof of Theorem 2 of Schoen-Simon SS81 goes as follows. By the successive blow-up argument, one arrives at a varifold $W_{l}$ which is a stationary, integral, codimension 1 cone in $\mathbb{R}^{n-l+1}$ such that $\operatorname{sing}\left(W_{l}\right)=\{0\}$ and

$$
\mathbb{R}^{l} \times W_{l}=\lim _{m \rightarrow \infty} J_{\#} \circ \tau_{y_{m} \#} \circ \mu_{r_{m} \#}\left|\Sigma_{q_{m}}\right|
$$

for some sequence of points $y_{m} \in \mathbb{R}^{n+1}$ and positive real numbers $r_{m}$ and some subsequence $\left\{\Sigma_{q_{m}}\right\} \subset\left\{\Sigma_{q}\right\} ;\left|y_{m}\right|, r_{m} \rightarrow \infty$. Here $J$ is some orthogonal transformation of $\mathbb{R}^{n+1}, \tau_{y}$ denotes the translation of $\mathbb{R}^{n+1}$ which brings $y$ to the origin, $\mu_{r}$ is the multiplication (scaling) by $r$. It is shown that (equation (5.22) of Schoen-Simon) $l \geq s$ for every $s$ such that $\mathcal{H}^{s}\left(\operatorname{sing}(\Sigma) \cap B^{n+1}\left(0, \frac{1}{2}\right)\right)>0$ (hence, one only needs to show that $l \leq n-7$ ) and $l \leq n-3$. Upto this point, the only facts about $\Sigma_{q}$ which are used : $\Sigma_{q}$ satisfies the stability inequality (1.17) and Theorem 1 of Schoen-Simon [SS81. After this, stability of $\left|\Sigma_{q}\right|$ is used to conclude that $\mathbb{R}^{l} \times W_{l}$ is stable. Hence, $W_{l}$ is a smooth, stable codimension 1 cone in $\mathbb{R}^{n-l+1}$ with a singularity at origin. Therefore, $n-l \geq 7$ i.e. $l \leq n-7$.

In our context of Theorem 4.3 the above mentioned proof can be modified as follows. As noted above, stability inequality (1.17) and Theorem 1 of SchoenSimon SS81] continue to hold under the weaker assumption $\lambda_{1}\left(\left|\Sigma_{q}\right|\right) \geq-\alpha$. Moreover

$$
\lambda_{1}\left(\left|\Sigma_{q}\right|\right) \geq-\alpha \Longrightarrow \lambda_{1}\left(J_{\#} \circ \tau_{y_{m} \#} \circ \mu_{r_{m} \#}\left|\Sigma_{q_{m}}\right|\right) \geq \frac{-\alpha}{r_{m}}
$$

4.3 can be justified, for example, using Theorem 3.5. If $\lambda_{1}\left(\mathbb{R}^{l} \times W_{l}\right)<0$ then $\lambda_{1}\left(\mathbb{R}^{l} \times W_{l}\right)<-\varepsilon$ for some $\varepsilon>0$. (This can be seen from the proof of Theorem 3.5.) Since $\mathbb{R}^{l} \times W_{l}$ is the varifold limit of $J_{\#} \circ \tau_{y_{m} \#} \circ \mu_{r_{m} \#}\left|\Sigma_{q_{m}}\right|$, in view of Remark 3.3, for all large $m$,

$$
\lambda_{1}\left(J_{\#} \circ \tau_{y_{m} \#} \circ \mu_{r_{m} \#}\left|\Sigma_{q_{m}}\right|\right)<-\varepsilon
$$

This contradicts 4.3 as $\lim _{m \rightarrow \infty} r_{m}=\infty$. Hence, $\lambda_{1}\left(\mathbb{R}^{l} \times W_{l}\right) \geq 0$ i.e. $\mathbb{R}^{l} \times W_{l}$ is stable. 
To prove the graphical convergence part of Theorem 1.2 we will need the following Lemma which is a consequence of Theorem 4.1 .

Lemma 4.4. Let $\left(N^{n+1}, g\right)$ be a closed Riemannian manifold. Let $\left\{\Sigma_{q}\right\}$ be a sequence of singular, minimal hypersurfaces in $N$ with $\mathcal{H}^{n-2}\left(\operatorname{sing}\left(\Sigma_{q}\right)\right)=0$ for all $q$. We also assume that $W_{q}=\left|\Sigma_{q}\right|$ varifold converges to a stationary, integral varifold $W, \Sigma=\operatorname{spt}(W)$ and $\Sigma_{q}$ converges to $\Sigma$ in the Hausdorff topology. Then, for every $x_{0} \in \operatorname{reg}(\Sigma)$ there exists $R=R\left(x_{0}\right)>0$ such that if $r \leq R$ and $\lambda_{1}\left(W_{q}\left\llcorner B^{N}\left(x_{0}, r\right)\right) \geq-\alpha\right.$ for all $q$ sufficiently large then $\Sigma_{q} \cap B^{N}\left(x_{0}, r / 4\right)$ is smooth for large $q$ and $\Sigma_{q}$ converges to $\Sigma$ smoothly in the ball $B^{N}\left(x_{0}, r / 5\right)$.

Proof. Let $\Theta=\lim _{t \rightarrow 0} \frac{\|W\|\left(B^{N}\left(x_{0}, t\right)\right)}{t^{n}}$. (The limit exists since $W$ is stationary.) Let $\rho_{0}$ be such that

$$
\frac{\|W\|\left(B^{N}\left(x_{0}, t\right)\right)}{t^{n}} \leq \Theta+1 / 2 \quad \forall t \leq \rho_{0}
$$

Let $\delta_{0}$ and $r_{0}$ be the constants which are provided by Theorem 4.1 when we set $\mu=\Theta+1$ and $\mu_{1}=1$. Let $s_{0}=\frac{\delta_{0} r_{0}}{2}$. For $0<a<\frac{1}{2} \operatorname{inj}_{N}$, identifying $\mathbb{R}^{n+1}$ with $T_{x_{0}} N$ (and $\mathbb{R}^{n} \times\{0\} \subset \mathbb{R}^{n+1}$ is identified with $T_{x_{0}} M$ ) we define

$$
\Phi_{a}: B^{n+1}(0,2) \longrightarrow B^{N}\left(x_{0}, 2 a\right), \quad \Phi_{a}(v)=\exp _{x_{0}}(a v), \quad g_{a}=\Phi_{a}^{*} g
$$

We can choose $R>0$ so that

$$
\text { - } R<\min \left\{\frac{1}{2} \operatorname{inj}_{N}, \frac{1}{2} d^{N}\left(x_{0}, \operatorname{sing}(\Sigma)\right), 1, \rho_{0}\right\}
$$

and whenever $r \leq R$

- $\left(B^{n+1}(0,1), g_{r}\right)$ satisfies 4.1 with $\mu_{1}=1$

- $\tilde{\Sigma}=\Phi_{r}^{-1}\left(\Sigma \cap B^{N}\left(x_{0}, 2 r\right)\right) \subset B^{n+1}(0,2) \cap\left\{x:\left|x_{n+1}\right|<s_{0}\right\} 2$

We will show that the above choice of $R=R\left(x_{0}\right)$ works. Let us fix an $r \leq R$. We define

$$
C=B^{n}(0,1) \times[-1,1] \subset B^{n+1}(0,2), \quad C^{\prime}=C \backslash\left\{x:\left|x_{n+1}\right|<s_{0}\right\} .
$$

Then

$$
C^{\prime} \cap\left(\tilde{\Sigma} \cup \partial B^{n+1}(0,2)\right)=\emptyset ; \quad d:=\operatorname{dist}_{g_{r}}\left(C^{\prime},\left(\tilde{\Sigma} \cup \partial B^{n+1}(0,2)\right)\right)
$$

We choose $q_{0}$ (depending on $r$ ) so that for all $q \geq q_{0}$

- $0<\frac{\left\|W_{q}\right\|\left(B^{N}\left(x_{0}, r\right)\right)}{r^{n}} \leq \Theta+13$

- $\lambda_{1}\left(W_{q}\left\llcorner B^{N}\left(x_{0}, r\right)\right) \geq-\alpha\right.$

- The Hausdorff distance $d_{H}\left(\Sigma_{q}, \Sigma\right)<d r$

\footnotetext{
${ }^{2}$ This is possible since $\Sigma$ is smooth near $x_{0}$

${ }^{3}$ This is possible because of 4.4 and varifold convergence of $W_{q}$ to $W$
} 
Hence, denoting $\tilde{\Sigma}_{q}=\Phi_{r}^{-1}\left(\Sigma_{q} \cap B^{N}\left(x_{0}, 2 r\right)\right)$, for all $q \geq q_{0}$ we have $\tilde{\Sigma}_{q} \cap$ $B^{n+1}(0,1) \neq \emptyset$,

$$
\begin{gathered}
\mathcal{H}^{n}\left(\tilde{\Sigma}_{q} \cap B^{n+1}(0,1)\right)=\frac{\mathcal{H}^{n}\left(\Sigma_{q} \cap B^{N}\left(x_{0}, r\right)\right)}{r^{n}} \leq \Theta+1, \\
\lambda_{1}\left(\left|\tilde{\Sigma}_{q} \cap B^{n+1}(0,1)\right|\right) \geq-\alpha r \geq-\alpha
\end{gathered}
$$

and

$$
\tilde{\Sigma}_{q} \cap C^{\prime}=\emptyset \quad \text { i.e. } \quad\left(\tilde{\Sigma}_{q} \cap C\right) \subset C \cap\left\{x:\left|x_{n+1}\right|<s_{0}\right\} 4
$$

We can now apply Theorem 4.1 to the singular, minimal hypersurface

$$
\left(\tilde{\Sigma}_{q} \cap B^{n+1}(0,1)\right) \subset\left(B^{n+1}(0,1), g_{r}\right)
$$

for $q \geq q_{0}$. Since, $B^{n+1}(0,1) \subset C$, equation 4.5 implies that for all $q \geq q_{0}$, $x \in \tilde{\Sigma}_{q} \cap B^{n+1}(0,1 / 4)$ and for $\rho=r_{0}$ the oscillation bound $(*)$ of Theorem 4.1 is satisfied in the cylinder $C\left(x, r_{0}\right)$. Hence, $\tilde{\Sigma}_{q} \cap B^{n+1}(0,1 / 4)$ is smooth with uniform bound on $A_{\tilde{\Sigma}_{q} \cap B^{n+1}(0,1 / 4)}$ and we have already noted above a uniform upper bound on $\mathcal{H}^{n}\left(\tilde{\Sigma}_{q} \cap B^{n+1}(0,1)\right)$. The counterpart of the ArzelaAscoli theorem for smooth, minimal hypersurface gives that in a smaller ball $B^{n+1}(0,1 / 5), \tilde{\Sigma}_{q}$ converges to $\tilde{\Sigma}$ smoothly and graphically. (Here we do not have to pass to a further subsequence since we already know that $\tilde{\Sigma}_{q}$ Hausdorff converges to $\tilde{\Sigma})$. When we rescale back to go back to $N$, we get that in the ball $B^{N}\left(x_{0}, r / 5\right), \Sigma_{q}$ converges to $\Sigma$ smoothly and graphically.

\section{Proof of the main theorem}

In this section we will give a proof of Theorem 1.2 .

By Allard's compactness theorem All72, possibly after passing to a subsequence, $V_{k} \longrightarrow V$ in the $\mathbf{F}$ metric with $V$ is a stationary, integral varifold and $\|V\|(N) \leq \Lambda$. If $\lambda_{p}(V)<-\alpha$ then $\lambda_{p}\left(V_{k}\right)<-\alpha$ for $k$ sufficiently large by Remark 3.3. Hence, $\lambda_{p}(V) \geq-\alpha$. We also know that $\operatorname{spt}\left(V_{k}\right)=M_{k}$ converges to $\operatorname{spt}(V)=M$ in the Hausdorff topology.

Lemma 5.1. Let $W$ be a stationary varifold in $U^{n+1}$ with $\lambda_{p}(W) \geq-\alpha, \alpha \geq 0$. Let $U_{1}, \ldots, U_{p}$ be mutually disjoint open subsets of $U$ such that $\|W\|\left(U_{j}\right) \neq 0$ for each $j=1, \ldots, p$. Then there exists $i \in\{1, \ldots, p\}$ such that $\lambda_{1}\left(W\left\llcorner U_{i}\right) \geq-\alpha\right.$.

Proof. Let us assume that for all $i=1, \ldots, p$ we have $\lambda_{1}\left(W\left\llcorner U_{i}\right)<-\alpha\right.$. Hence, from Definition 3.2 and equation 3.1 we have maps

$$
F^{i}: \bar{B}^{1} \longrightarrow \operatorname{Diff}\left(U_{i}\right)
$$

\footnotetext{
${ }^{4}$ This follows from $d_{H}\left(\Sigma_{q}, \Sigma\right)<d r$
} 
and vector-fields $Y^{i}$ compactly supported in $U_{i}$

$$
\left.Y^{i}\right|_{x}=\left.\frac{d}{d t}\right|_{0} F_{t}^{i}(x)
$$

such that

$$
\delta^{2} W\left(Y^{i}, Y^{i}\right)<-\alpha\left\|Y^{i}\right\|_{L^{2}}^{2}
$$

We define

$$
F: \bar{B}^{p} \longrightarrow \operatorname{Diff}(U), \quad F_{v}=\Phi^{\sum_{i} v_{i} Y^{i}}
$$

where $\Phi^{X}$ denotes the time 1 flow of the vector field $X$. For this choice of $F$ we have

$$
\begin{aligned}
\left.D^{2} A^{W}\right|_{0}(u, u) & =\delta^{2} W\left(\sum_{i} u_{i} Y^{i}, \sum_{i} u_{i} Y^{i}\right) \\
& =\sum_{i} u_{i}^{2} \delta^{2} W\left(Y^{i}, Y^{i}\right) \quad\left(\operatorname{since} \operatorname{spt}\left(Y^{i}\right)^{\prime} \text { s are mutually disjoint }\right) \\
& <-\alpha \sum_{i} u_{i}^{2}\left\|Y^{i}\right\|_{L^{2}}^{2} \\
& =-\alpha\left\|\sum_{i} u_{i} Y^{i}\right\|_{L^{2}}^{2} \quad \text { (since } \operatorname{spt}\left(Y^{i}\right) \text { 's are mutually disjoint) } \\
& =-\alpha K^{W}(u, u)
\end{aligned}
$$

This contradicts $\lambda_{p}(W) \geq-\alpha$.

Returning back to the proof of the main Theorem 1.2, let $G \subset M$ be the set of points $x \in M$ for which there exists $r=r(x)>0$ and some subsequence $\left\{V_{k^{\prime}}\right\} \subset\left\{V_{k}\right\}$ such that for each $k^{\prime}, \lambda_{1}\left(V_{k^{\prime}}\left\llcorner B^{N}(x, 2 r)\right) \geq-\alpha\right.$. Therefore, using Theorem 4.3, $\mathcal{H}^{s}\left(\operatorname{sing}(M) \cap B^{N}(x, r(x))\right)=0$ for all $x \in G$ and $s>n-7$.

Lemma 5.2. The set $M \backslash G$ has atmost $p-1$ points.

Proof. Suppose there exists $p$ points $\left\{x_{i}\right\}_{i=1}^{p} \subset M \backslash G$. Let $t$ be small enough so that the normal geodesic balls $\left\{B^{N}\left(x_{i}, t\right)\right\}_{i=1}^{p}$ are mutually disjoint. By the definition of $G$, there exists $k_{0}$ such that for all $k \geq k_{0}, \lambda_{1}\left(V_{k}\left\llcorner B^{N}\left(x_{i}, t\right)\right)<\right.$ $-\alpha$ for each $i$. By Lemma 5.1, this implies $\lambda_{p}\left(V_{k}\right)<-\alpha$ for all $k \geq k_{0}$, a contradiction.

We note that

$$
\operatorname{sing}(M) \cap G=\bigcup_{x \in \sin g(M) \cap G}\left(\operatorname{sing}(M) \cap B^{N}(x, r(x))\right)
$$

We can extract a countable subcover of the R.H.S. of 5.1 and writ $e^{5}$

$$
\operatorname{sing}(M) \cap G=\bigcup_{i=1}^{\infty}\left(\operatorname{sing}(M) \cap B^{N}\left(x_{i}, r\left(x_{i}\right)\right)\right)
$$

\footnotetext{
${ }^{5}$ This is possible because $N$, being a manifold, is second-countable and a subspace of a second-countable space is second-countable.
} 
where each $x_{i}$ is in $\operatorname{sing}(M) \cap G$. By the definition of $G$, for $s>n-7$, $\mathcal{H}^{s}\left(\operatorname{sing}(M) \cap B^{N}\left(x_{i}, r\left(x_{i}\right)\right)\right)=0$ for each $i$. Therefore, 5.2 and Lemma 5.2 imply that $\mathcal{H}^{s}(\operatorname{sing}(M))=0$. (Here we are implicitly assuming that $n \geq 7$ so that for $s>n-7 \geq 0, \mathcal{H}^{s}(M \backslash G)=0$; when $n<7$ further arguments are required to show that $M$ is smooth at the points of $M \backslash G$ as explained in ACS16.)

We can now complete the proof of graphical convergence. We will produce a set $\mathcal{Y} \subset \operatorname{reg}(M)$ and a subsequence $\left\{M_{k^{\prime}}\right\} \subset\left\{M_{k}\right\}$ such that $M_{k^{\prime}}$ converges smoothly and graphically on compact subsets of $\operatorname{reg}(M) \backslash \mathcal{Y}$. Let $X$ be a countable, dense subset of $\operatorname{reg}(M)$ and

$$
\mathcal{B}=\left\{B^{N}(x, r): x \in X, r \in \mathbb{Q}^{+}, r<d^{N}(x, \operatorname{sing}(M)), r<\operatorname{inj}_{N}\right\}
$$

Then $\mathcal{B}$ is a countable collection of balls, say, $\mathcal{B}=\left\{B_{i}\right\}_{i=1}^{\infty}$. We will mark each $B_{i}$ as good or bad and to each $B_{i}$ we will assign an infinite index set $I_{i} \subset \mathbb{N}$ as follows. At the first step we examine whether there exists an infinite set $J \subset \mathbb{N}$ such that $\left\{M_{j}\right\}_{j \in J}$ converges to $M$ smoothly in $B_{1}$. If it exists we mark $B_{1}$ as good and define $I_{1}$ to be that $J$. Otherwise we mark $B_{1}$ as bad and define $I_{1}$ to be $\mathbb{N}$. Suppose we have marked $B_{i-1}$ as good or bad and defined $I_{i-1}$. Then we examine whether there exists an infinite set $J \subset I_{i-1}$ such that $\left\{M_{j}\right\}_{j \in J}$ converges to $M$ smoothly in $B_{i}$. If it exists we mark $B_{i}$ as good and define $I_{i}$ to be that $J$. Otherwise we mark $B_{i}$ as bad and define $I_{i}$ to be $I_{i-1}$.

Let $\mathcal{G}$ be the union of good balls. Denoting $\mathcal{Y}=\operatorname{reg}(M) \backslash \mathcal{G}$, we claim that $|\mathcal{Y}| \leq p-1$. Otherwise, there exists $p$ distinct points $x_{1}, \ldots, x_{p}$ in $\mathcal{Y}$. To arrive at a contradiction we will apply Lemma 4.4 to the sequence of singular, minimal hypersurfaces $\left\{M_{k}\right\} ;\left|M_{k}\right|$ converges in the varifold sense to $V$ which is supported on $M$; Lemma 4.4 provides a function $R: \operatorname{reg}(M) \longrightarrow \mathbb{R}^{+}$. Let $\tau$ be a positive number such that $5 \tau \leq R\left(x_{l}\right)$ for each $l$ and the balls $\left\{B^{N}\left(x_{l}, 5 \tau\right)\right\}_{l=1}^{p}$ are mutually disjoint. There exists $B_{i_{l}} \in \mathcal{B}$ such that $x_{l} \in B_{i_{l}} \subset B^{N}\left(x_{l}, \tau\right)$. As $x_{l} \in \mathcal{Y}, B_{i_{l}}$ is a bad ball. Hence $\left\{M_{j}\right\}_{j \in I_{i_{l}}}$ does not have a subsequence which smoothly converges to $M$ in $B^{N}\left(x_{l}, \tau\right)$. Therefore, by Lemma 4.4, $\lambda_{1}\left(V_{j}\left\llcorner B^{N}\left(x_{l}, 5 \tau\right)\right)<-\alpha\right.$ for all large $j \in I_{i_{l}}$. Without loss of generality, we can assume that $i_{1}<\ldots<i_{p}$ so that $I_{i_{1}} \supset \ldots \supset I_{i_{p}}$. Hence $\lambda_{1}\left(V_{j}\left\llcorner B^{N}\left(x_{l}, 5 \tau\right)\right)<-\alpha\right.$ for each $l=1, . ., p$ and for all large $j \in I_{i_{p}}$. By Lemma 5.1 this gives $\lambda_{p}\left(V_{j}\right)<-\alpha$ for all large $j \in I_{i_{p}}$, a contradiction. Hence, $|\mathcal{Y}| \leq p-1$.

By a diagonal argument, we can choose an infinite set $I \subset \mathbb{N}$ such that $\left|I \backslash I_{i}\right|$ is finite for all $i$. Then, by the definition of good ball, $\left\{M_{i}\right\}_{i \in I}$ is a sequence which converges smoothly and graphically on the compact subsets of $\operatorname{reg}(M) \cap \mathcal{G}=$ $\operatorname{reg}(M) \backslash \mathcal{Y}$.

\section{Some further remarks}

Besides the main compactness Theorems of [Sha17] and ACS16], some additional results proved in these two papers can be suitably generalized in higher 
dimensions. In this last section, we will state them as a sequence of remarks. Below we will assume that $M_{k}$ 's and $M$ are as in Theorem 1.2 (and Theorem 1.3).

Remark 6.1. We have assumed that each $M_{k}$ is connected. Since $\left\{M_{k}\right\}$ converges to $M$ in the Hausdorff distance, this implies that $M$ is connected as well. From [Ilm96] (Theorem A (ii)) it follows that reg $(M)$ and hence $\operatorname{reg}(M) \backslash \mathcal{Y}$ is also connected. Therefore, the number of sheets in the graphical convergence is constant over $\operatorname{reg}(M) \backslash \mathcal{Y}$. In particular, $V=m|M|$ for some $m \in \mathbb{N}$.

Remark 6.2. If the number of sheets in the graphical convergence is 1 , then $\mathcal{Y}=\emptyset$. This is Claim 4 in Sha17 and the proof presented there works in our case as well.

Remark 6.3. Suppose $\operatorname{reg}(M)$ is two sided. If the number of sheets in the graphical convergence is at least 2 or if the number of sheets is 1 and $M_{k} \cap M=\emptyset$ for large $k$, we can construct a positive Jacobi field on $\operatorname{reg}(M)$. In this case, $\operatorname{reg}(M)$ and hence $M$ is stable. The proof is same as presented in Sha17.

Remark 6.4. Continuing with Remark 6.3 suppose $\operatorname{Ric}(N, g)>0$. Then the convergence of $M_{k}$ to $M$ is always single sheeted. This can be thought of as a higher dimensional analogue of Choi-Schoen CS85] which asserts that in a three manifold with possitive Ricci curvature, the space of closed, embedded minimal surfaces with bounded genus is compact in the smooth topology. Indeed, in our case, if $H_{n}\left(N, \mathbb{Z}_{2}\right)=0$ then $\operatorname{reg}(M)$ is two sided; hence, if the number of sheets is $\geq 2, M$ is stable by the above Remark 6.3. However, as proved in Zho17. (Lemma 2.8) positive Ricci curvature of $(N, g)$ implies that $M$ can not be stable. The general case can be obtained by lifting $M_{k}$ 's and $M$ to the universal cover $\tilde{N}$ of $N$ (by [Fra66] and Lemma 2.10 of [Zho17] the lifts $\tilde{M}_{k}, \tilde{M}$ are connected).

Remark 6.5. Theorems 1.2 and 1.3 hold in the varying metric set-up. More precisely, instead of assuming $\left|M_{k}\right|$ is stationary with respect to the fixed metric $g$ if we assume that $\left|M_{k}\right|$ is stationary with respect to the metric $g_{k}$ and $g_{k}$ converges to $g$ in $C^{3}$, Theorems 1.2 and 1.3 continue to hold.

\section{References}

[ACS16] L. Ambrozio, A. Carlotto, and B. Sharp, Compactness of the space of minimal hypersurfaces with bounded volume and p-th jacobi eigenvalue, J. Geom. Anal. 4 (2016), 2591-2601.

[All72] W. K. Allard, On the first variation of a varifold, Ann. of Math. 95(3) (1972), 417-491.

[Alm65] F. Almgren, The theory of varifolds, Mimeographed notes, Princeton, 1965.

[CM18] O. Chodosh and C. Mantoulidis, Minimal surfaces and the allen-cahn equation on 3-manifolds: index, multiplicity, and curvature estimates, arXiv:1803.02716 [math.DG] (2018).

[CS85] H. I. Choi and R. Schoen, The space of minimal embeddings of a surface into a threedimensional manifold of positive ricci curvature, Invent. Math. 81 (1985), 387-394. 
[Fra66] T. Frankel, On the fundamental group of a compact minimal submanifold, Ann. of Math. 83 (1) (1966), 68-73.

[Gas17] Pedro Gaspar, The second inner variation of energy and the morse index of limit interfaces, arXiv:1710.04719 [math.DG] (2017).

[GG18] P. Gaspar and M. A. M. Guaraco, The weyl law for the phase transition spectrum and the density of minimal hypersurfaces, arXiv:1804.04243 [math.DG] (2018).

[Hie17] F. Hiesmayr, Spectrum and index of two-sided allen-cahn minimal hypersurfaces, arXiv:1704.07738 [math.DG] (2017).

[Ilm96] T. Ilmanen, A strong maximum principle for singular minimal hypersurfaces, Calc. Var. 4 (1996), 443-467.

[IMN18] K. Irie, F. C. Marques, and A. Neves, Density of minimal hypersurfaces for generic metrics, Ann. of Math. 187 (2018), 963-972.

[Li19] Y. Li, Existence of infinitely many minimal hypersurfaces in higher-dimensional closed manifolds with generic metrics, arXiv:1901.08440 [math.DG] (2019).

[LMN18] Y. Liokumovich, F. C. Marques, and A. Neves, Weyl law for the volume spectrum, Ann. of Math. 187 (2018), 933-961.

[MN17] F. C. Marques and A. Neves, Existence of infinitely many minimal hypersurfaces in positive ricci curvature, Invent. Math. 209 (2017), no. 2, 577-616.

[MN16] _ Morse index and multiplicity of min-max minimal hypersurfaces, Cambridge J. Math. 4 (2016), no. 4, 463-511.

[MNS17] F. C. Marques, A. Neves, and A. Song, Equidistribution of minimal hypersurfaces in generic metrics, arXiv:1712.06238 [math.DG] (2017).

[Pit81] J. Pitts, Existence and regularity of minimal surfaces on riemannian manifolds, Mathematical Notes 27, Princeton University Press, Princeton, 1981.

[Sha17] B. Sharp, Compactness of minimal hypersurfaces with bounded index, J. Differential Geom. 106 (2017), 317-339.

[Sim] L. Simon, Lectures on geometric measure theory. Proceedings of the Centre for Mathematical Analysis, Australian National University, Canberra, (1983).

[Son18] A. Song, Existence of infinitely many minimal hypersurfaces in closed manifolds, arXiv:1806.08816 [math.DG] (2018).

[SS81] R. Schoen and L. Simon, Regularity of stable minimal hypersurfaces, Comm. Pure Appl. Math. 34 (1981), 741-797.

[Whi17] B. White, On the bumpy metrics theorem for minimal submanifolds, Amer. J. Math 139(4) (2017), 1149-1155.

[Whi91] _ The space of minimal submanifolds for varying riemannian metrics, Indiana Univ. Math. J. 40 (1991), 161-200.

[Zho17] X. Zhou, Min-max hypersurface in manifold of positive ricci curvature, J. Differential Geom. 105 (2017), no. 2, 291-343.

[Zho19] On the multiplicity one conjecture in min-max theory, arXiv:1901.01173 [math.DG] (2019). 